\title{
Genome-wide association study of high-altitude pulmonary edema in a Han Chinese population
}

\author{
Xun Li, ${ }^{1, *}$ Tianbo Jin ${ }^{2,3, *}$, Mingxia Zhang ${ }^{3}$, Hua Yang ${ }^{3}$, Xuewen Huang ${ }^{1}$, Xiaobo \\ Zhou ${ }^{1}$, Wenchao Huang ${ }^{1}$, Lipeng Qin ${ }^{1}$, Longli Kang ${ }^{2}$, Ming Fan ${ }^{4}$, Suzhi Li ${ }^{1}$ \\ ${ }^{1}$ Center of Altitude Disease, General Hospital of Tibet Military Area Command, Lhasa 850003, China \\ ${ }^{2}$ Key Laboratory of High Altitude Environment and Genes Related to Diseases of Tibet Autonomous Region, School of \\ Medicine, Xizang Minzu University, Xianyang, Shaanxi 712082, China \\ ${ }^{3}$ School of Life Sciences, Northwest University, Xi'an, Shaanxi 710069, China \\ ${ }^{4}$ Department of Brain Protection and Plasticity, Institute of Basic Medical Sciences, Beijing 100850, China \\ *These authors contributed equally to this work and are joint first authors \\ Correspondence to: Ming Fan, email: fanming1973@126.com \\ Suzhi Li, email: suzhilixizang@163.com
}

Keywords: high-altitude pulmonary edema (HAPE), single nucleotide polymorphisms (SNPS), genome wide association analysis (GWAS), susceptibility gene

Received: October 20, $2016 \quad$ Accepted: February 27, $2017 \quad$ Published: March 18, 2017

Copyright: Li et al. This is an open-access article distributed under the terms of the Creative Commons Attribution License (CC-BY), which permits unrestricted use, distribution, and reproduction in any medium, provided the original author and source are credited.

\section{ABSTRACT}

A two-stage genome-wide association study (GWAS) was performed to identify and analyze genes and single nucleotide polymorphisms (SNPs) associated with high-altitude pulmonary edema (HAPE) in a Han Chinese patient population. In the first stage, DNA samples from 68 patients with recurrent HAPE were scanned using Affymetrix SNP Array 6.0 Chips, and allele frequencies were compared to those of 84 HapMap CHB samples to identify candidate SNPs. In the second stage, the 77 identified candidate SNPs were examined in an independent cohort of samples from 199 HAPE patients and 304 controls. Associations between SNPs and HAPE risk were tested using various genetic models. Of the 77 original SNPs, 7 were found to be associated with HAPE susceptibility in the second stage of the study. GO and pathway enrichment analysis of the 7 SNPs revealed 5 adjacent genes involved in various processes, including regulation of nucleoside diphosphate metabolism, thyroid hormone catabolism, and low-density lipoprotein receptor activity. These results suggest the identified SNPs and genes may contribute to the physiopathology of HAPE.

\section{INTRODUCTION}

High altitude pulmonary edema (HAPE) is a noncardiogenic form of pulmonary edema that develops in unacclimatized healthy individuals at altitudes above $2500-3000 \mathrm{~m}$ [1]. It is a potentially fatal medical condition and the most common cause of death among high-altitude illnesses [2]. However, the pathogenesis of HAPE remains poorly understood. Previous studies suggest that uneven hypoxic pulmonary vasoconstriction, pulmonary capillary damage, and increased pulmonary artery pressure play important roles in the pathogenesis of HAPE $[3,4]$.

HAPE is caused by the interaction of both genetic and environmental risk factors. Previous studies have shown that family history and race influence individual susceptibilities to HAPE [5]. Some people are susceptible to high-altitude pulmonary edema, whereas others are resistant to this condition $[6,7]$. The prevalence of HAPE in the Han Chinese population in Tibet, which is about $0.4 \% \sim 2 \%$ [8] and differs depending on age, gender, and occupation, is higher than that observed in native Tibetans. Rate of ascent, altitude reached, pre-acclimatization, and individual susceptibility are the major factors that contribute to high-altitude maladies [9]. In addition, patients who have previously developed HAPE are more likely to experience recurrence, which suggests the presence of a constitutional, and possibly a genetic, component in its etiology [10].

Several recent studies have examined the genetic basis of HAPE, focusing mainly on genetic polymorphisms 
in the beta2-adrenergic receptor [11], vascular endothelial growth factor [12], the renin angiotensin system [13], and pulmonary surfactant proteins A1 and A2 [14] in subjects susceptible to HAPE. Polymorphisms within these genes may explain individual variation in hypoxic responses and perhaps indicate susceptibility to high-altitude disease. However, the precise role of these genes in HAPE pathogenesis remains unclear.

To identify genetic variants across the whole genome that are specifically related to HAPE risk, we conducted a two-stage GWAS analysis in 68 patients with recurrent HAPE and in 84 HapMap CHB populations as references. We further evaluated potential associations with HAPE risk in a replication cohort with a total of 199 HAPE patients and 304 healthy controls from a Han Chinese population. While previous GWAS studies were based on case-control samples only, here we examined a large number of cases to identify genes that might be related to HAPE susceptibility.

\section{RESULTS}

A total of 571 subjects, including 267 HAPE patients (246 males, 21 females; mean age $32.6 \pm 10.7$ ) and 304 controls (290 males, 14 females; mean age 36.2 \pm 4.5 ), were examined in this analysis. Age distribution differed between the patient and control groups $(p<0.05)$. Participant characteristics are listed in Table 1.

We first scanned DNA samples from 68 patients with recurrent HAPE using Affymetrix Genome-Wide Human SNP Array 6.0 Chips. After filtering with standard quality-control procedures, 502,689 SNPs with an overall call rate of $99.92 \%$ were qualified for further GWAS analysis. To identify SNPs that might be associated with the risk of HAPE, we compared SNP allele frequencies in the 68 patients to those of the 84 HapMap CHB controls and found that frequencies differed for 77 SNPs. Information regarding these 77 SNPs and their associated genes is shown in Table 2. A Manhattan plot was generated for the SNPs in patients with recurrent HAPE under the allelic and genotypic model (Figure 1). MDS and QQ-plot revealed that there was no obvious population stratification in this experiment (Figure 2 and Figure 3).

Of the 77 SNPs, 68 were qualified after Sequenom MassARRAY Assay Design. In a second experiment, we confirmed the results of the first experiment by genotyping the 68 SNPs in 199 HAPE patients and 304 controls of Han Chinese descent. Table 3 summarizes the characteristics of the tested SNPs and their predicted associations with HAPE risk in crude analysis. Three SNPs (rs17484974, rs725050, and rs10178082) were excluded at the $5 \% p$-value for Hardy-Weinberg equilibrium (HWE). A $\chi^{2}$ test revealed that two SNPs, rs 10789097 (OR = 1.825; $95 \% \mathrm{CI}=1.062-3.135, p=0.027)$, and $\mathrm{rs} 17777329$ $(\mathrm{OR}=1.800 ; 95 \% \mathrm{CI}=1.083-2.991, p=0.022)$ were associated with an increased risk of HAPE (Table 3).
Associations between the SNPs and HAPE risk were tested under five different genetic models (co-dominant, dominant, co-dominant, recessive, and log-additive). Seven SNPs were associated with HAPE susceptibility. The rs41417552 SNP was associated with an increased risk of HAPE based on the results of the co-dominant $(\mathrm{OR}=1.58$; $95 \% \mathrm{CI}=1.04-2.40, p=0.057$ for the "A/G" genotype), dominant $(\mathrm{OR}=1.62 ; 95 \% \mathrm{CI}=1.07-2.44, p=0.022$ for the "A/G-G/G" genotype), over-dominant (OR = 1.87; $95 \% \mathrm{CI}=1.06-3.27, p=0.03$ for the "A/G" genotype), and log-additive $(\mathrm{OR}=1.59 ; 95 \% \mathrm{CI}=1.09-2.32, p=0.017)$ models. The rs10984811 SNP increased HAPE risk in both the co-dominant $(\mathrm{OR}=3.95 ; 95 \% \mathrm{CI}=1.33-11.73$, $p=0.032$ for the " $\mathrm{C} / \mathrm{C}$ " genotype) and recessive $(\mathrm{OR}=3.97 ; 95 \% \mathrm{CI}=1.34-11.75, p=0.0089$ for the "C/C" genotype) models. The rs17777329 SNP was also associated with an increased risk of HAPE in the codominant $(\mathrm{OR}=1.88 ; 95 \% \mathrm{CI}=1.07-3.30, p=0.051)$, dominant $(\mathrm{OR}=1.95 ; 95 \% \mathrm{CI}=1.12-3.37, p=0.018)$, over-dominant $(\mathrm{OR}=1.87 ; 95 \% \mathrm{CI}=1.06-3.27, p=0.03)$, and log-additive $(\mathrm{OR}=1.89 ; 95 \% \mathrm{CI}=1.13-3.16$, $p=0.015)$ models. The rs 1075355 SNP was associated with increased HAPE risk in the co-dominant $(\mathrm{OR}=1.65$; 95\% CI $=1.04-2.62, p=0.093)$ and over-dominant $(\mathrm{OR}=1.66 ; 95 \% \mathrm{CI}=1.04-2.63, p=0.032)$ models. Additionally, the rs12226072 (OR $=0.58 ; 95 \%$ $\mathrm{CI}=0.40-0.86, p=0.0053)$ and $\mathrm{rs} 6074799(\mathrm{OR}=0.59$; $95 \% \mathrm{CI}=0.37-0.93, p=0.02)$ SNPs were associated with a decreased risk of HAPE in the over-dominant model, and the rs7677143 SNP was associated with a decreased risk of HAPE in the log-additive model (OR $=0.69 ; 95 \%$ $\mathrm{CI}=0.48-0.99, p=0.039$ ) (Table 4).

To identify genes that might be involved in HAPE invasion, we also performed gene annotation and functional classification for the 7 significant loci we identified in the replication study. GO and KEGG pathway enrichment analyses identified 5 potential candidate genes located within $\pm 500 \mathrm{~kb}$ of these SNPs (Table 5). These genes were mainly involved in cellular tight junctions, oxidation and reduction, extracellular matrix metabolism, pulmonary development, and pulmonary smooth muscular tension adjustment.

\section{DISCUSSION}

In this study, we conducted a two-stage GWAS analysis to investigate the genetic factors associated with HAPE in a Han Chinese population. Seven loci, including four susceptibility loci and three protective loci, were found to be associated with HAPE in this analysis. Gene annotation and functional classification of these loci revealed that five of the candidate genes are potentially involved in the pathogenesis of HAPE. To the best of our knowledge, this is one of the largest studies to explore the genetic factors underlying the development of HAPE in a Han Chinese population. 
Table 1: Basic characteristics of cases and controls in this study

\begin{tabular}{lccc}
\hline \multicolumn{1}{c}{ Variables } & Case N (\%) & Control N (\%) & $p$-value \\
\hline Age (years) & $32.6 \pm 10.7$ & $36.2 \pm 4.5$ & $<0.005^{\text {a }}$ \\
Sex & & & $>0.005^{\text {b }}$ \\
Male & $246(92.0 \%)$ & $290(95.4 \%$ & \\
Female & $21(8.0 \%)$ & $14(4.6 \%)$ & \\
Total & 267 & 304 & \\
\hline
\end{tabular}

${ }^{a} P$ values were calculated from two-sided chi-square tests.

${ }^{\mathrm{b}} P$ values were calculated by Student $t$ tests.

The rs10789097 locus contained no annotated genes, and the gene nearest to it was INADL, which encodes inactivation no-after potential (INAD) protein, also known as protein associated with Lin seven 1 (Pals1) -associated tight junction protein (PATJ). INAD contains multiple PDZ domains, which are protein-protein interaction modules that typically bind to short peptide sequences at the carboxyl terminus of target proteins. Proteins containing multiple PDZ domains often bind to different transmembrane and intracellular proteins and play central roles as organizers of multimeric complexes [15]. PATJ is a polarity protein and plays a complex role in the maintenance of epithelial polarity [16]. Considering that stress failure in pulmonary capillaries is an important contributor to HAPE pathogenesis, we speculate that the INADL gene may also impact the pathogenesis of HAPE.

The KCNV2 gene, which encodes the Kcnv2 protein, belongs to a group of potassium channel
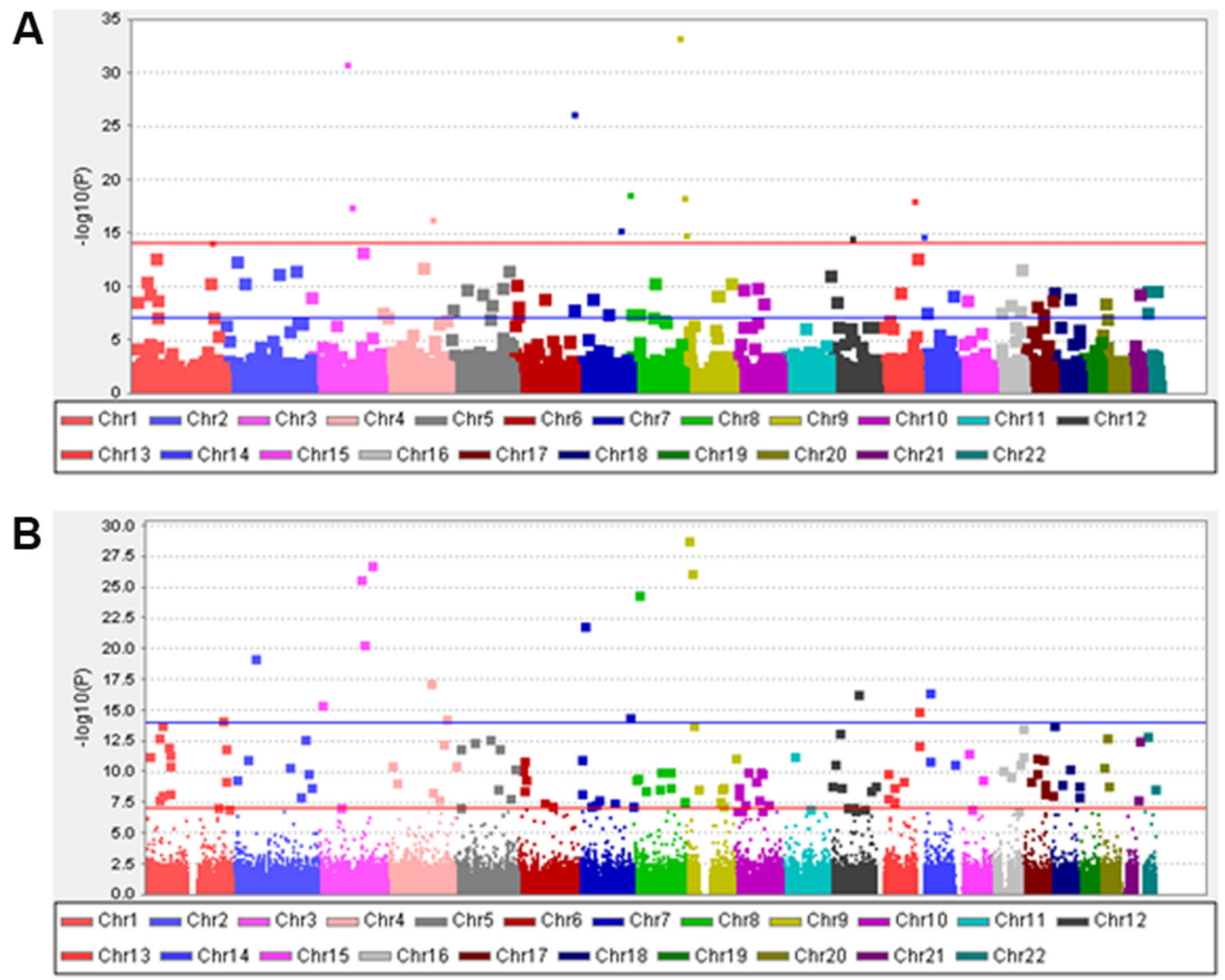

Figure 1: Manhattan plot for the whole SNPs in recurrent HAPE subjects of Chinese Han decent. Chromosomes are shown in alternate colors. (A) Allelic model; (B) Genotypic model. 
Table 2: Basic information of the significantly different SNPs between 68 recurrent HAPE cases and 84 Hapmap CHB subjects in the first stage

\begin{tabular}{|c|c|c|c|c|c|c|c|c|}
\hline \multirow{2}{*}{ SNP ID } & \multirow{2}{*}{ Chromosome } & \multirow{2}{*}{ Gene (s) } & \multirow{2}{*}{$\frac{\text { Alleles }}{\mathbf{A}^{\mathrm{a} / \mathbf{B}}}$} & \multicolumn{2}{|c|}{ MAF } & \multirow{2}{*}{ Position } & \multirow{2}{*}{ Band } & \multirow{2}{*}{ Role } \\
\hline & & & & Case & Control & & & \\
\hline rs4908427 & 1 & CAMTA1 & $\mathrm{G} / \mathrm{A}$ & 0.059 & 0.054 & 6976226 & $1 \mathrm{p} 36.31$ & Intron \\
\hline rs9661274 & 1 & & $\mathrm{G} / \mathrm{A}$ & 0.059 & 0.060 & 30149249 & $1 \mathrm{p} 35.3$ & \\
\hline rs17484974 & 1 & & $\mathrm{C} / \mathrm{T}$ & 0.132 & 0.054 & 39186794 & $1 \mathrm{p} 34.3$ & \\
\hline rs 12406517 & 1 & PPAP2B & $\mathrm{G} / \mathrm{C}$ & 0.110 & 0.072 & 56974278 & $1 \mathrm{p} 32.2$ & Intron \\
\hline rs1694212 & 1 & & $\mathrm{~T} / \mathrm{C}$ & 0.132 & 0.113 & 61480000 & $1 \mathrm{p} 31.3$ & \\
\hline rs10789097 & 1 & & $\mathrm{C} / \mathrm{G}$ & 0.066 & 0.071 & 62119978 & $1 \mathrm{p} 31.3$ & \\
\hline rs17188846 & 1 & KCNH1 & $\mathrm{C} / \mathrm{G}$ & 0.184 & 0.067 & 211261821 & $1 \mathrm{q} 32.2$ & Intron \\
\hline rs2577156 & 1 & EPRS & $\mathrm{C} / \mathrm{A}$ & 0.051 & 0.077 & 220190845 & $1 \mathrm{q} 41$ & Intron \\
\hline rs3008613 & 1 & MIA3 & $\mathrm{G} / \mathrm{A}$ & 0.110 & 0.079 & 222795769 & $1 \mathrm{q} 41$ & Intron \\
\hline rs4491711 & 2 & RASGRP3 & $\mathrm{G} / \mathrm{A}$ & 0.103 & 0.066 & 33776743 & $2 \mathrm{p} 22.3$ & Intron \\
\hline rs11125567 & 2 & CCDC88A & $\mathrm{A} / \mathrm{G}$ & 0.081 & 0.143 & 55627913 & $2 \mathrm{p} 16.1$ & Intron \\
\hline rs11898268 & 2 & & $\mathrm{C} / \mathrm{A}$ & 0.000 & 0.071 & 154622125 & $2 q 23.3$ & \\
\hline rs 10167840 & 2 & & $\mathrm{~T} / \mathrm{G}$ & 0.140 & 0.077 & 199241493 & $2 \mathrm{q} 33.1$ & \\
\hline rs7612512 & 3 & & $\mathrm{G} / \mathrm{C}$ & 0.140 & 0.157 & 3412838 & $3 \mathrm{p} 26.2$ & \\
\hline rs1846594 & 3 & & $\mathrm{C} / \mathrm{T}$ & 0.213 & 0.196 & 112916203 & $3 q 13.2$ & \\
\hline rs11924340 & 3 & & $\mathrm{~A} / \mathrm{G}$ & 0.060 & 0.110 & 145325196 & $3 q 24$ & \\
\hline rs 12504325 & 4 & C4orf6 & $\mathrm{A} / \mathrm{G}$ & 0.103 & 0.083 & 5537184 & $4 \mathrm{p} 16.2$ & Downstream \\
\hline rs17598758 & 4 & & $\mathrm{G} / \mathrm{T}$ & 0.110 & 0.089 & 20190068 & $4 \mathrm{p} 15.31$ & \\
\hline rs7677143 & 4 & & $\mathrm{C} / \mathrm{T}$ & 0.147 & 0.220 & 117082198 & $4 q 26$ & \\
\hline rs6535838 & 4 & & $\mathrm{~A} / \mathrm{C}$ & 0.199 & 0.101 & 153023402 & $4 q 31.3$ & \\
\hline rs7688505 & 4 & & $\mathrm{~T} / \mathrm{A}$ & 0.110 & 0.110 & 185828318 & $4 q 35.1$ & \\
\hline rs41417552 & 5 & CMBL & $\mathrm{G} / \mathrm{A}$ & 0.110 & 0.083 & 10305452 & $5 \mathrm{p} 15.2$ & Intron \\
\hline rs2161592 & 5 & & $\mathrm{~A} / \mathrm{G}$ & 0.162 & 0.085 & 50772554 & $5 q 11.2$ & \\
\hline rs3777207 & 5 & ELL2 & $\mathrm{A} / \mathrm{G}$ & 0.118 & 0.084 & 95231115 & $5 q 15$ & Intron \\
\hline rs6595114 & 5 & & $\mathrm{C} / \mathrm{T}$ & 0.118 & 0.101 & 117676709 & $5 q 23.1$ & \\
\hline rs2193963 & 5 & & $\mathrm{C} / \mathrm{T}$ & 0.096 & 0.089 & 121596196 & $5 \mathrm{q} 23.2$ & \\
\hline rs17652561 & 5 & SLC6A7 & $\mathrm{A} / \mathrm{G}$ & 0.162 & 0.185 & 149584197 & $5 q 32$ & $\begin{array}{c}\text { Intron } \\
\text { (boundary) }\end{array}$ \\
\hline rs2937582 & 5 & & $\mathrm{~A} / \mathrm{G}$ & 0.434 & 0.080 & 166465008 & $5 q 34$ & \\
\hline rs2984100 & 6 & & $\mathrm{C} / \mathrm{G}$ & 0.125 & 0.143 & 8592499 & $6 \mathrm{p} 24.3$ & \\
\hline rs7762263 & 6 & & $\mathrm{~T} / \mathrm{C}$ & 0.110 & 0.066 & 11975250 & $6 \mathrm{p} 24.1$ & \\
\hline rs4715938 & 6 & & $\mathrm{G} / \mathrm{C}$ & 0.103 & 0.113 & 14944857 & $6 \mathrm{p} 23$ & \\
\hline rs725050 & 6 & & $\mathrm{C} / \mathrm{T}$ & 0.162 & 0.196 & 89267376 & $6 q 15$ & \\
\hline rs1419722 & 7 & EIF3B & $\mathrm{C} / \mathrm{T}$ & 0.142 & 0.107 & 2413258 & $7 \mathrm{p} 22.3$ & Intron \\
\hline rs10178082 & 7 & & $\mathrm{~T} / \mathrm{A}$ & 0.199 & 0.157 & 10706912 & $7 \mathrm{p} 21.3$ & \\
\hline rs4947936 & 7 & & $\mathrm{C} / \mathrm{A}$ & 0.103 & 0.133 & 50906752 & $7 \mathrm{p} 12.1$ & \\
\hline rs 12226072 & 7 & & $\mathrm{~A} / \mathrm{T}$ & 0.294 & 0.339 & 96443614 & $7 \mathrm{q} 21.3$ & \\
\hline rs2956956 & 8 & DLGAP2 & $\mathrm{C} / \mathrm{T}$ & 0.066 & 0.083 & 1553118 & $8 \mathrm{p} 23.3$ & Intron \\
\hline rs2980508 & 8 & SGK223 & $\mathrm{C} / \mathrm{T}$ & 0.096 & 0.106 & 8171732 & $8 \mathrm{p} 23.1$ & Downstream \\
\hline rs310282 & 8 & & $\mathrm{C} / \mathrm{A}$ & 0.132 & 0.125 & 23614369 & $8 \mathrm{p} 21.2$ & \\
\hline rs4573320 & 8 & & $\mathrm{C} / \mathrm{T}$ & 0.343 & 0.446 & 65128758 & $8 \mathrm{q} 12.3$ & \\
\hline rs1568828 & 8 & PREX2 & $\mathrm{A} / \mathrm{G}$ & 0.081 & 0.101 & 69122128 & $8 \mathrm{q} 13.2$ & Intron \\
\hline rs 1006698 & 9 & KCNV2 & $\mathrm{T} / \mathrm{G}$ & 0.206 & 0.232 & 2725283 & $9 \mathrm{p} 24.2$ & Intron \\
\hline rs1011531 & 9 & & $\mathrm{~A} / \mathrm{G}$ & 0.110 & 0.114 & 13755192 & 9 p23 & \\
\hline rs13289064 & 9 & & $\mathrm{C} / \mathrm{G}$ & 0.228 & 0.179 & 16897685 & $9 \mathrm{p} 22.2$ & \\
\hline rs10984811 & 9 & ANP32B & $\mathrm{C} / \mathrm{A}$ & 0.149 & 0.173 & 100784050 & $9 q 22.33$ & Downstream \\
\hline
\end{tabular}




\begin{tabular}{|c|c|c|c|c|c|c|c|c|}
\hline rs 12554842 & 9 & COL5A1 & $\mathrm{T} / \mathrm{C}$ & 0.081 & 0.071 & 137573407 & $9 \mathrm{q} 34.3$ & Intron \\
\hline rs11593009 & 10 & & $\mathrm{~T} / \mathrm{A}$ & 0.051 & 0.065 & 31974946 & $10 \mathrm{p} 11.22$ & \\
\hline rs 12243354 & 10 & TET1 & $\mathrm{A} / \mathrm{G}$ & 0.125 & 0.131 & 70411536 & $10 \mathrm{q} 21.3$ & $\begin{array}{c}\text { Intron } \\
\text { (boundary) }\end{array}$ \\
\hline rs7923700 & 10 & GRID1 & $\mathrm{G} / \mathrm{A}$ & 0.162 & 0.190 & 87843290 & $10 \mathrm{q} 23.1$ & Intron \\
\hline rs2239153 & 12 & VWF & $\mathrm{C} / \mathrm{T}$ & 0.338 & 0.399 & 6186667 & $12 \mathrm{p} 13.31$ & Intron \\
\hline rs7303062 & 12 & & $\mathrm{~A} / \mathrm{G}$ & 0.074 & 0.084 & 22990450 & $12 \mathrm{p} 12.1$ & \\
\hline rs10879780 & 12 & & $\mathrm{~T} / \mathrm{G}$ & 0.235 & 0.226 & 74837984 & $12 \mathrm{q} 21.1$ & \\
\hline rs1316571 & 13 & & $\mathrm{~T} / \mathrm{C}$ & 0.081 & 0.095 & 68320718 & $13 \mathrm{q} 21.32$ & \\
\hline rs9550256 & 13 & FAM70B & $\mathrm{A} / \mathrm{T}$ & 0.265 & 0.220 & 114494675 & $13 q 34$ & Intron \\
\hline rs17435983 & 14 & & $\mathrm{~A} / \mathrm{G}$ & 0.169 & 0.101 & 27860597 & $14 \mathrm{q} 12$ & \\
\hline rs8007744 & 14 & & $\mathrm{G} / \mathrm{A}$ & 0.265 & 0.262 & 28329396 & $14 q 12$ & \\
\hline rs17777329 & 14 & & G/A & 0.081 & 0.060 & 101934762 & $14 q 32.31$ & \\
\hline rs4787426 & 16 & IL4R & $\mathrm{G} / \mathrm{T}$ & 0.059 & 0.065 & 27384731 & 16 p12.1 & Downstream \\
\hline rs 1075355 & 16 & VAT1L & $\mathrm{C} / \mathrm{G}$ & 0.147 & 0.107 & 77874149 & $16 \mathrm{q} 23.1$ & Intron \\
\hline rs12931468 & 16 & ATP2C2 & $\mathrm{G} / \mathrm{C}$ & 0.074 & 0.054 & 84495301 & $16 q 24.1$ & $\begin{array}{c}\text { Intron } \\
\text { (boundary) }\end{array}$ \\
\hline rs8067836 & 17 & LASP1 & $\mathrm{G} / \mathrm{T}$ & 0.081 & 0.071 & 37081707 & 17q12 & Downstream \\
\hline rs16955841 & 17 & HLF & $\mathrm{G} / \mathrm{A}$ & 0.105 & 0.107 & 53364146 & $17 q 22$ & Intron \\
\hline rs 12450240 & 17 & NARF & $\mathrm{T} / \mathrm{G}$ & 0.265 & 0.235 & 80423712 & $17 q 25.3$ & Intron \\
\hline rs9961715 & 18 & DLGAP1 & $\mathrm{C} / \mathrm{T}$ & 0.029 & 0.054 & 3824312 & $18 p 11.31$ & Intron \\
\hline rs12606093 & 18 & KIAA0427 & $\mathrm{C} / \mathrm{A}$ & 0.044 & 0.065 & 46077295 & $18 q 21.1$ & Intron \\
\hline rs6074799 & 20 & MACROD2 & $\mathrm{G} / \mathrm{C}$ & 0.110 & 0.101 & 14771472 & $20 p 12.1$ & Intron \\
\hline rs9617661 & 22 & TUBA8 & $\mathrm{G} / \mathrm{T}$ & 0.029 & 0.060 & 18595352 & $22 q 11.21$ & Intron \\
\hline rs5758913 & 22 & & $\mathrm{C} / \mathrm{T}$ & 0.154 & 0.161 & 43148259 & $22 \mathrm{q} 13.2$ & \\
\hline
\end{tabular}

Notes: A/B stands for minor/major alleles on the entire sample frequencies.

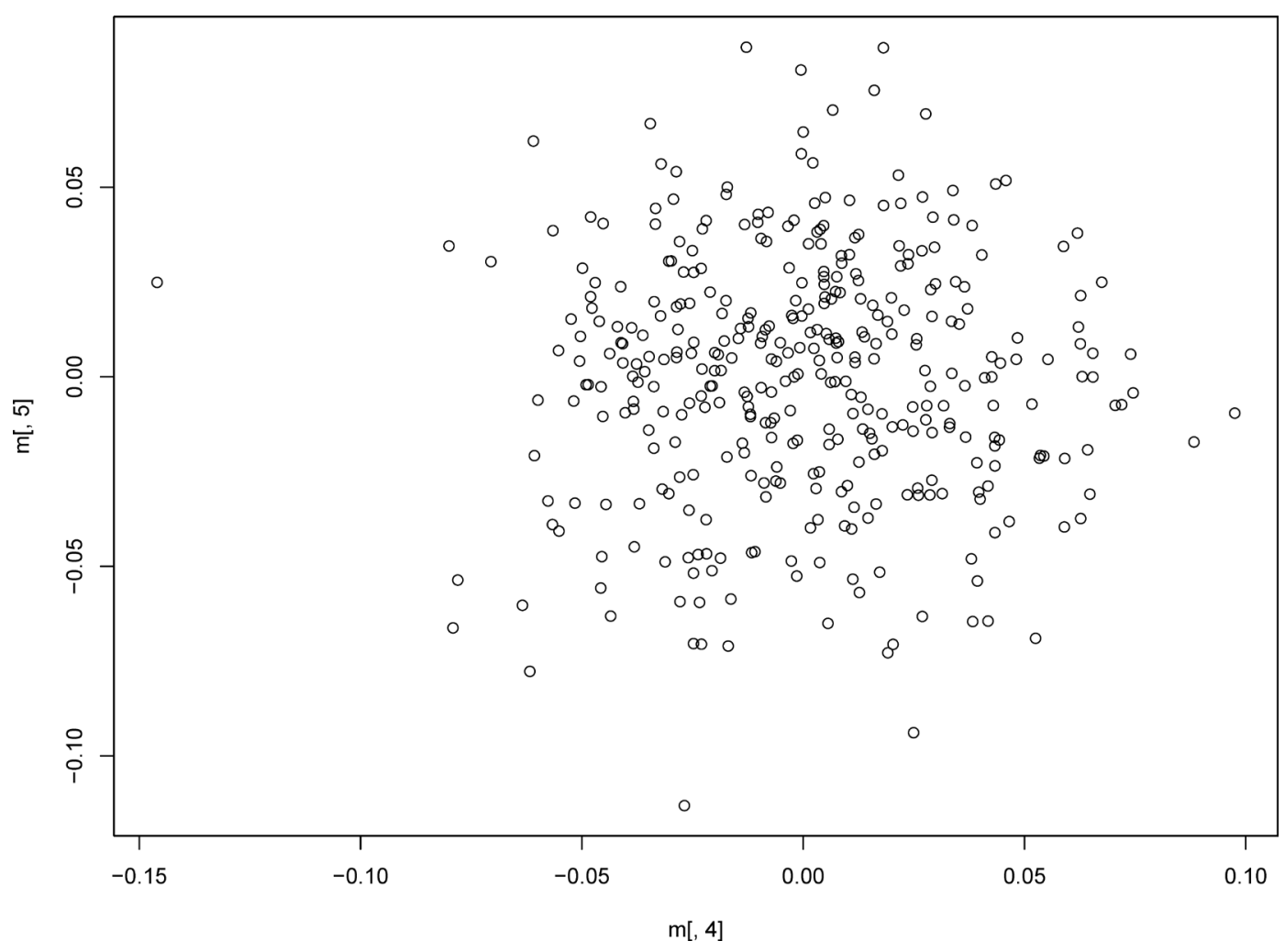

Figure 2: Multidimensional scaling approach (MDS) analysis for the first stage. 
Table 3: Allele frequencies in cases and controls and odds ratio estimates for HAPE for the replication stage

\begin{tabular}{|c|c|c|c|c|c|c|c|c|c|}
\hline \multirow{3}{*}{$\frac{\text { SNP ID }}{\text { rs4908427 }}$} & \multirow{3}{*}{$\begin{array}{l}\text { Gene (s) } \\
\text { CAMTA1 }\end{array}$} & \multirow{3}{*}{$\begin{array}{c}\text { Alleles } \\
\mathbf{A}^{\mathrm{a} / \mathbf{B}} \\
\mathrm{G} / \mathrm{A}\end{array}$} & \multicolumn{2}{|c|}{ MAF } & \multirow{2}{*}{$\frac{\text { HWE }}{p \text {-value }}$} & \multirow{2}{*}{ ORs } & \multirow{2}{*}{\multicolumn{2}{|c|}{$95 \%$ CI }} & \multirow{3}{*}{$\frac{\boldsymbol{p} \text {-value }}{0.337}$} \\
\hline & & & \multirow{2}{*}{$\frac{\text { Case }}{0.035}$} & \multirow{2}{*}{$\frac{\text { Control }}{0.048}$} & & & & & \\
\hline & & & & & 1 & 0.728 & 0.380 & 1.395 & \\
\hline rs9661274 & & $\mathrm{G} / \mathrm{A}$ & 0.065 & 0.067 & 0.3787 & 0.967 & 0.581 & 1.607 & 0.896 \\
\hline rs17484974 & & $\mathrm{C} / \mathrm{T}$ & 0.111 & 0.112 & $7.851 \mathrm{E}-47^{\#}$ & 0.987 & 0.660 & 1.476 & 0.949 \\
\hline rs 12406517 & PPAP2B & $\mathrm{G} / \mathrm{C}$ & 0.055 & 0.054 & 0.2131 & 1.020 & 0.585 & 1.776 & 0.946 \\
\hline rs1694212 & & $\mathrm{T} / \mathrm{C}$ & 0.139 & 0.133 & 0.8025 & 1.049 & 0.726 & 1.517 & 0.798 \\
\hline rs10789097 & & $\mathrm{C} / \mathrm{G}$ & 0.075 & 0.043 & 1 & 1.825 & 1.062 & 3.135 & $0.027 *$ \\
\hline rs17188846 & KCNH1 & $\mathrm{C} / \mathrm{G}$ & 0.139 & 0.130 & 0.198 & 1.080 & 0.746 & 1.564 & 0.683 \\
\hline rs2577156 & EPRS & $\mathrm{C} / \mathrm{A}$ & 0.076 & 0.077 & 0.08963 & 0.978 & 0.607 & 1.576 & 0.928 \\
\hline rs3008613 & MIA3 & $\mathrm{G} / \mathrm{A}$ & 0.093 & 0.105 & 0.552 & 0.871 & 0.569 & 1.334 & 0.526 \\
\hline rs4491711 & RASGRP3 & $\mathrm{G} / \mathrm{A}$ & 0.063 & 0.097 & 1 & 0.624 & 0.384 & 1.014 & 0.055 \\
\hline rs11125567 & CCDC88A & $\mathrm{A} / \mathrm{G}$ & 0.111 & 0.127 & 1 & 0.857 & 0.578 & 1.271 & 0.443 \\
\hline rs11898268 & & $\mathrm{C} / \mathrm{A}$ & 0.003 & 0.000 & 1 & - & - & - & - \\
\hline rs 10167840 & & $\mathrm{~T} / \mathrm{G}$ & 0.088 & 0.095 & 1 & 0.914 & 0.589 & 1.420 & 0.690 \\
\hline rs7612512 & & $\mathrm{G} / \mathrm{C}$ & 0.151 & 0.172 & 1 & 0.857 & 0.606 & 1.212 & 0.382 \\
\hline rs 1846594 & & $\mathrm{C} / \mathrm{T}$ & 0.193 & 0.232 & 0.6287 & 0.794 & 0.582 & 1.085 & 0.148 \\
\hline rs 11924340 & & $\mathrm{~A} / \mathrm{G}$ & 0.111 & 0.086 & 0.4683 & 1.329 & 0.871 & 2.029 & 0.186 \\
\hline rs 12504325 & C4orf6 & $\mathrm{A} / \mathrm{G}$ & 0.083 & 0.090 & 0.4886 & 0.909 & 0.579 & 1.428 & 0.679 \\
\hline rs 17598758 & & $\mathrm{G} / \mathrm{T}$ & 0.101 & 0.105 & 1 & 0.950 & 0.626 & 1.441 & 0.808 \\
\hline rs7677143 & & $\mathrm{C} / \mathrm{T}$ & 0.143 & 0.191 & 1 & 0.709 & 0.502 & 1.002 & 0.051 \\
\hline rs6535838 & & $\mathrm{A} / \mathrm{C}$ & 0.133 & 0.137 & 0.4691 & 0.972 & 0.671 & 1.407 & 0.879 \\
\hline rs7688505 & & $\mathrm{T} / \mathrm{A}$ & 0.156 & 0.140 & 0.8133 & 1.135 & 0.796 & 1.619 & 0.483 \\
\hline rs41417552 & CMBL & $\mathrm{G} / \mathrm{A}$ & 0.169 & 0.127 & 0.4419 & 1.404 & 0.985 & 2.003 & 0.060 \\
\hline rs2161592 & & $\mathrm{A} / \mathrm{G}$ & 0.108 & 0.102 & 0.7525 & 1.067 & 0.707 & 1.609 & 0.758 \\
\hline rs3777207 & ELL2 & $\mathrm{A} / \mathrm{G}$ & 0.108 & 0.107 & 0.5511 & 1.012 & 0.673 & 1.521 & 0.955 \\
\hline rs6595114 & & $\mathrm{C} / \mathrm{T}$ & 0.118 & 0.097 & 0.5042 & 1.246 & 0.830 & 1.870 & 0.288 \\
\hline rs2193963 & & $\mathrm{C} / \mathrm{T}$ & 0.106 & 0.095 & 1 & 1.130 & 0.742 & 1.721 & 0.568 \\
\hline rs17652561 & SLC6A7 & $\mathrm{A} / \mathrm{G}$ & 0.145 & 0.151 & 0.6541 & 0.949 & 0.663 & 1.357 & 0.773 \\
\hline rs2937582 & & $\mathrm{A} / \mathrm{G}$ & 0.439 & 0.434 & 0.6413 & 1.021 & 0.791 & 1.318 & 0.871 \\
\hline rs 2984100 & & $\mathrm{C} / \mathrm{G}$ & 0.184 & 0.156 & 0.2747 & 1.220 & 0.873 & 1.707 & 0.244 \\
\hline rs7762263 & & $\mathrm{T} / \mathrm{C}$ & 0.111 & 0.123 & 0.594 & 0.883 & 0.595 & 1.312 & 0.539 \\
\hline rs4715938 & & $\mathrm{G} / \mathrm{C}$ & 0.161 & 0.155 & 0.3873 & 1.048 & 0.741 & 1.481 & 0.792 \\
\hline rs 725050 & & $\mathrm{C} / \mathrm{T}$ & 0.249 & 0.243 & $0.04164^{\#}$ & 1.029 & 0.767 & 1.381 & 0.849 \\
\hline rs1419722 & EIF3B & $\mathrm{C} / \mathrm{T}$ & 0.143 & 0.149 & 0.648 & 0.958 & 0.669 & 1.372 & 0.816 \\
\hline rs10178082 & & $\mathrm{T} / \mathrm{A}$ & 0.141 & 0.161 & $0.0001595^{\#}$ & 0.852 & 0.597 & 1.216 & 0.378 \\
\hline rs4947936 & & $\mathrm{C} / \mathrm{A}$ & 0.163 & 0.150 & 0.6502 & 1.109 & 0.784 & 1.569 & 0.559 \\
\hline rs 12226072 & & $\mathrm{~A} / \mathrm{T}$ & 0.317 & 0.340 & 0.7994 & 0.897 & 0.685 & 1.175 & 0.431 \\
\hline rs2956956 & DLGAP2 & $\mathrm{C} / \mathrm{T}$ & 0.078 & 0.092 & 0.7288 & 0.833 & 0.527 & 1.317 & 0.433 \\
\hline rs2980508 & SGK223 & $\mathrm{C} / \mathrm{T}$ & 0.146 & 0.135 & 1 & 1.097 & 0.763 & 1.576 & 0.619 \\
\hline rs310282 & & $\mathrm{C} / \mathrm{A}$ & 0.096 & 0.135 & 0.3231 & 0.678 & 0.451 & 1.019 & 0.061 \\
\hline rs 4573320 & & $\mathrm{C} / \mathrm{T}$ & 0.279 & 0.299 & 0.8912 & 0.907 & 0.685 & 1.200 & 0.493 \\
\hline
\end{tabular}




\begin{tabular}{|c|c|c|c|c|c|c|c|c|c|}
\hline rs 1568828 & PREX2 & $\mathrm{A} / \mathrm{G}$ & 0.108 & 0.109 & 0.3729 & 0.995 & 0.662 & 1.494 & 0.980 \\
\hline rs 1006698 & KCNV2 & $\mathrm{T} / \mathrm{G}$ & 0.216 & 0.263 & 0.2387 & 0.772 & 0.572 & 1.041 & 0.089 \\
\hline rs1011531 & & $\mathrm{A} / \mathrm{G}$ & 0.118 & 0.120 & 1 & 0.981 & 0.664 & 1.450 & 0.925 \\
\hline rs 13289064 & & $\mathrm{C} / \mathrm{G}$ & 0.231 & 0.183 & 1 & 1.346 & 0.986 & 1.837 & 0.060 \\
\hline rs10984811 & ANP32B & $\mathrm{C} / \mathrm{A}$ & 0.178 & 0.148 & 0.6479 & 1.250 & 0.889 & 1.757 & 0.199 \\
\hline rs 12554842 & COL5A1 & $\mathrm{T} / \mathrm{C}$ & 0.095 & 0.109 & 0.2273 & 0.867 & 0.569 & 1.320 & 0.505 \\
\hline rs11593009 & & $\mathrm{T} / \mathrm{A}$ & 0.078 & 0.076 & 0.6833 & 1.032 & 0.642 & 1.658 & 0.896 \\
\hline rs 12243354 & TET1 & $\mathrm{A} / \mathrm{G}$ & 0.138 & 0.137 & 0.809 & 1.014 & 0.703 & 1.464 & 0.940 \\
\hline rs7923700 & GRID1 & $\mathrm{G} / \mathrm{A}$ & 0.116 & 0.117 & 0.399 & 0.988 & 0.666 & 1.467 & 0.954 \\
\hline rs 2239153 & VWF & $\mathrm{C} / \mathrm{T}$ & 0.415 & 0.428 & 0.4145 & 0.948 & 0.734 & 1.224 & 0.682 \\
\hline rs7303062 & & $\mathrm{A} / \mathrm{G}$ & 0.050 & 0.061 & 0.6129 & 0.817 & 0.467 & 1.428 & 0.477 \\
\hline rs 10879780 & & $\mathrm{~T} / \mathrm{G}$ & 0.193 & 0.192 & 0.5762 & 1.007 & 0.731 & 1.389 & 0.964 \\
\hline rs1316571 & & $\mathrm{T} / \mathrm{C}$ & 0.078 & 0.079 & 0.102 & 0.985 & 0.616 & 1.577 & 0.951 \\
\hline rs9550256 & FAM70B & $\mathrm{A} / \mathrm{T}$ & 0.234 & 0.261 & 0.232 & 0.865 & 0.644 & 1.161 & 0.333 \\
\hline rs17435983 & & $\mathrm{A} / \mathrm{G}$ & 0.143 & 0.128 & 0.7988 & 1.136 & 0.786 & 1.640 & 0.497 \\
\hline rs8007744 & & $\mathrm{G} / \mathrm{A}$ & 0.261 & 0.267 & 0.7692 & 0.973 & 0.729 & 1.299 & 0.852 \\
\hline rs17777329 & & $\mathrm{G} / \mathrm{A}$ & 0.085 & 0.049 & 0.5287 & 1.800 & 1.083 & 2.991 & $0.022 *$ \\
\hline rs4787426 & IL4R & $\mathrm{G} / \mathrm{T}$ & 0.083 & 0.066 & 1 & 1.284 & 0.795 & 2.073 & 0.306 \\
\hline rs1075355 & VAT1L & $\mathrm{C} / \mathrm{G}$ & 0.131 & 0.092 & 0.1553 & 1.481 & 0.992 & 2.212 & 0.053 \\
\hline rs 12931468 & ATP2C2 & $\mathrm{G} / \mathrm{C}$ & 0.055 & 0.044 & 1 & 1.259 & 0.707 & 2.244 & 0.434 \\
\hline rs8067836 & LASP1 & $\mathrm{G} / \mathrm{T}$ & 0.111 & 0.082 & 0.7057 & 1.387 & 0.906 & 2.125 & 0.131 \\
\hline rs16955841 & HLF & $\mathrm{G} / \mathrm{A}$ & 0.133 & 0.109 & 0.1143 & 1.255 & 0.830 & 1.898 & 0.282 \\
\hline rs 12450240 & NARF & $\mathrm{T} / \mathrm{G}$ & 0.242 & 0.281 & 0.8872 & 0.818 & 0.612 & 1.093 & 0.174 \\
\hline rs9961715 & DLGAP1 & $\mathrm{C} / \mathrm{T}$ & 0.055 & 0.041 & 0.4021 & 1.364 & 0.758 & 2.455 & 0.298 \\
\hline rs 12606093 & KIAA0427 & $\mathrm{C} / \mathrm{A}$ & 0.063 & 0.066 & 0.3739 & 0.952 & 0.568 & 1.595 & 0.851 \\
\hline rs6074799 & MACROD2 & $\mathrm{G} / \mathrm{C}$ & 0.113 & 0.140 & 0.2313 & 0.784 & 0.533 & 1.154 & 0.216 \\
\hline rs9617661 & TUBA8 & $\mathrm{G} / \mathrm{T}$ & 0.050 & 0.033 & 1 & 1.556 & 0.826 & 2.930 & 0.168 \\
\hline rs5758913 & & $\mathrm{C} / \mathrm{T}$ & 0.156 & 0.151 & 0.6541 & 1.035 & 0.729 & 1.469 & 0.848 \\
\hline
\end{tabular}

Notes: a Minor allele; ${ }^{*} p$ value $\leq 0.05$ indicates statistical significance; ${ }^{*}$ site with $\operatorname{HWE} p \leq 0.05$ is excluded;

Abbreviations: HWE, Hardy-Weinberg Equilibrium; MAF, minor allele frequency; SNP, single nucleotide polymorphism; ORs, odds ratios; $\mathrm{CI}$, confidence interval.

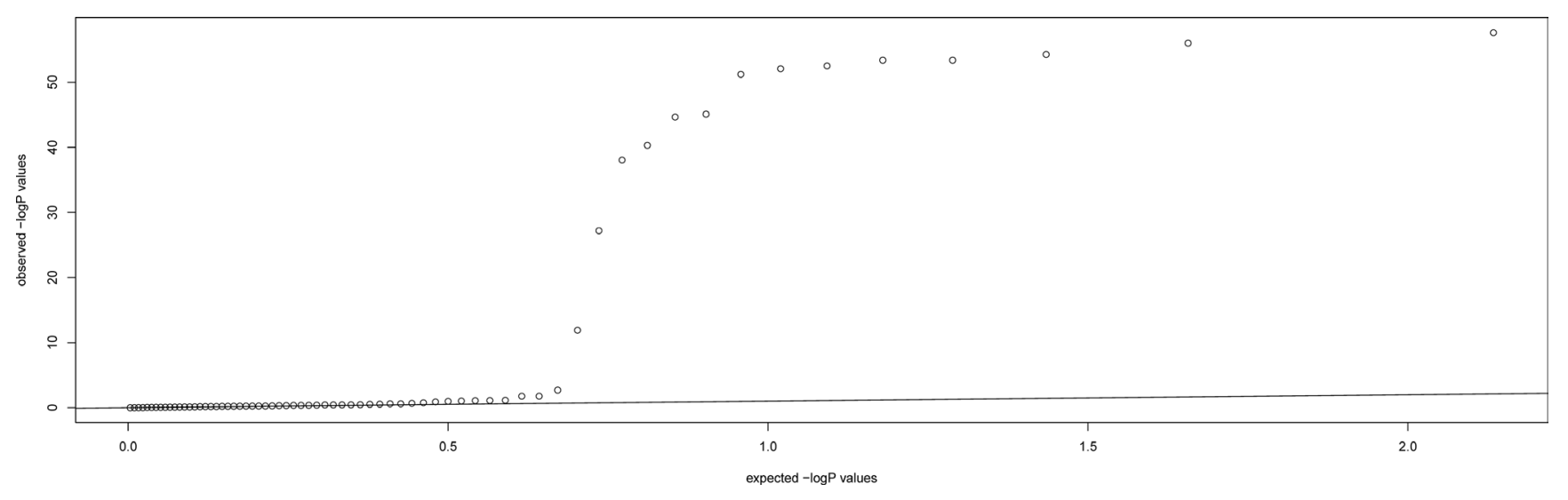

Figure 3: QQ plot for the whole SNPs for the first stage. 
Table 4: Logistic regression analysis of the associations between SNPs and HAPE risk

\begin{tabular}{|c|c|c|c|c|c|c|c|c|}
\hline SNP & Model & Genotype & Controls & Cases & OR $(95 \% \text { CI })^{a}$ & $P$-value ${ }^{\text {a }}$ & AIC & BIC \\
\hline \multirow[t]{10}{*}{ rs7677143 } & \multirow{3}{*}{ Co-dominant } & $\mathrm{T} / \mathrm{T}$ & $199(65.5 \%)$ & $145(72.9 \%)$ & 1 & \multirow{3}{*}{0.11} & \multirow{3}{*}{661.6} & \multirow{3}{*}{682.7} \\
\hline & & $\mathrm{T} / \mathrm{C}$ & $94(30.9 \%)$ & $51(25.6 \%)$ & $0.72(0.48-1.09)$ & & & \\
\hline & & $\mathrm{C} / \mathrm{C}$ & $11(3.6 \%)$ & $3(1.5 \%)$ & $0.38(0.10-1.40)$ & & & \\
\hline & \multirow{2}{*}{ Dominant } & $\mathrm{T} / \mathrm{T}$ & $199(65.5 \%)$ & $145(72.9 \%)$ & 1 & \multirow{2}{*}{0.062} & \multirow{2}{*}{660.6} & \multirow{2}{*}{677.5} \\
\hline & & $\mathrm{T} / \mathrm{C}-\mathrm{C} / \mathrm{C}$ & $105(34.5 \%)$ & $54(27.1 \%)$ & $0.69(0.46-1.02)$ & & & \\
\hline & \multirow{2}{*}{ Recessive } & $\mathrm{T} / \mathrm{T}-\mathrm{T} / \mathrm{C}$ & $293(96.4 \%)$ & $196(98.5 \%)$ & 1 & \multirow{2}{*}{0.16} & \multirow{2}{*}{662.1} & \multirow{2}{*}{678.9} \\
\hline & & $\mathrm{C} / \mathrm{C}$ & $11(3.6 \%)$ & $3(1.5 \%)$ & $0.41(0.11-1.53)$ & & & \\
\hline & \multirow{2}{*}{ Over-dominant } & $\mathrm{T} / \mathrm{T}-\mathrm{C} / \mathrm{C}$ & $210(69.1 \%)$ & $148(74.4 \%)$ & 1 & \multirow{2}{*}{0.16} & \multirow{2}{*}{662.1} & \multirow{2}{*}{678.9} \\
\hline & & $\mathrm{T} / \mathrm{C}$ & $94(30.9 \%)$ & $51(25.6 \%)$ & $0.75(0.50-1.12)$ & & & \\
\hline & Log-additive & - & - & - & $0.69(0.48-0.99)$ & 0.039 & 659.8 & 676.7 \\
\hline \multirow[t]{10}{*}{ rs 12226072} & \multirow{3}{*}{ Co-dominant } & $\mathrm{T} / \mathrm{T}$ & $131(43.1 \%)$ & $103(51.8 \%)$ & 1 & \multirow{3}{*}{0.017} & \multirow{3}{*}{657.9} & \multirow{3}{*}{679} \\
\hline & & $\mathrm{A} / \mathrm{T}$ & $139(45.7 \%)$ & $66(33.2 \%)$ & $0.61(0.41-0.90)$ & & & \\
\hline & & $\mathrm{A} / \mathrm{A}$ & $34(11.2 \%)$ & $30(15.1 \%)$ & $1.19(0.67-2.09)$ & & & \\
\hline & \multirow{2}{*}{ Dominant } & $\mathrm{T} / \mathrm{T}$ & $131(43.1 \%)$ & $103(51.8 \%)$ & 1 & & & \\
\hline & & $\mathrm{A} / \mathrm{T}-\mathrm{A} / \mathrm{A}$ & $173(56.9 \%)$ & $96(48.2 \%)$ & $0.72(0.50-1.04)$ & 0.071 & 000.9 & $0 / 1.0$ \\
\hline & Recescive & $\mathrm{T} / \mathrm{T}-\mathrm{A} / \mathrm{T}$ & $270(88.8 \%)$ & $169(84.9 \%)$ & 1 & 016 & 662 & 6780 \\
\hline & hecessive the & $\mathrm{A} / \mathrm{A}$ & $34(11.2 \%)$ & $30(15.1 \%)$ & $1.48(0.86-2.54)$ & 0.10 & 002 & 070.9 \\
\hline & Oyer dominant & T/T-A/A & $165(54.3 \%)$ & $133(66.8 \%)$ & 1 & 00052 & 6562 & 6722 \\
\hline & ver-dommant & $\mathrm{A} / \mathrm{T}$ & $139(45.7 \%)$ & $66(33.2 \%)$ & $0.58(0.40-0.86)$ & 0.0055 & 050.3 & 015.2 \\
\hline & Log-additive & - & - & - & $0.92(0.71-1.20)$ & 0.56 & 663.7 & 680.6 \\
\hline rs6074799 & & $\mathrm{C} / \mathrm{C}$ & $222(73 \%)$ & $159(79.9 \%)$ & 1 & & & \\
\hline & Co-dominant & $\mathrm{C} / \mathrm{G}$ & $79(26 \%)$ & $35(17.6 \%)$ & $0.60(0.38-0.95)$ & 0.03 & 659 & 680.1 \\
\hline & & $\mathrm{G} / \mathrm{G}$ & $3(1 \%)$ & $5(2.5 \%)$ & $2.57(0.59-11.13)$ & & & \\
\hline & Dominant & $\mathrm{C} / \mathrm{C}$ & $222(73 \%)$ & $159(79.9 \%)$ & 1 & 0068 & 6607 & 6776 \\
\hline & DOUntint & $\mathrm{C} / \mathrm{G}-\mathrm{G} / \mathrm{G}$ & $82(27 \%)$ & $40(20.1 \%)$ & $0.67(0.43-1.04)$ & 0.000 & 000.1 & 071.0 \\
\hline & Receccive & $\mathrm{C} / \mathrm{C}-\mathrm{C} / \mathrm{G}$ & $301(99 \%)$ & $194(97.5 \%)$ & 1 & 015 & 662 & 6780 \\
\hline & Kecessive & $\mathrm{G} / \mathrm{G}$ & $3(1 \%)$ & $5(2.5 \%)$ & $2.88(0.67-12.40)$ & 0.13 & 002 & $0 / 0.9$ \\
\hline & Oyer dominant & $\mathrm{C} / \mathrm{C}-\mathrm{G} / \mathrm{G}$ & $225(74 \%)$ & $164(82.4 \%)$ & 1 & 002 & 6587 & 6756 \\
\hline & Uver-dominant & $\mathrm{C} / \mathrm{G}$ & $79(26 \%)$ & $35(17.6 \%)$ & $0.59(0.37-0.93)$ & 0.02 & 058.1 & $0 / 5.0$ \\
\hline & Log-additive & - & - & - & $0.78(0.52-1.15)$ & 0.21 & 662.5 & 679.4 \\
\hline rs41417552 & & $\mathrm{A} / \mathrm{A}$ & $230(75.7 \%)$ & $135(68.2 \%)$ & 1 & & & \\
\hline & Co-dominant & $\mathrm{A} / \mathrm{G}$ & $71(23.4 \%)$ & $59(29.8 \%)$ & $1.58(1.04-2.40)$ & 0.057 & 658.3 & 679.4 \\
\hline & & $\mathrm{G} / \mathrm{G}$ & $3(1 \%)$ & $4(2 \%)$ & $2.68(0.58-12.38)$ & & & \\
\hline & Dominant & $\mathrm{A} / \mathrm{A}$ & $230(75.7 \%)$ & $135(68.2 \%)$ & 1 & 0020 & 6567 & 6736 \\
\hline & Domminat & $\mathrm{A} / \mathrm{G}-\mathrm{G} / \mathrm{G}$ & $74(24.3 \%)$ & $63(31.8 \%)$ & $1.62(1.07-2.44)$ & 0.022 & 050.1 & $0 / 3.0$ \\
\hline & Perecrive & $\mathrm{A} / \mathrm{A}-\mathrm{A} / \mathrm{G}$ & $301(99 \%)$ & $194(98 \%)$ & 1 & 027 & 6608 & 6777 \\
\hline & Kecessive & $\mathrm{G} / \mathrm{G}$ & $3(1 \%)$ & $4(2 \%)$ & $2.35(0.51-10.80)$ & 0.21 & 660.8 & $6 / 1.1$ \\
\hline & Over-dominant & $\mathrm{A} / \mathrm{A}-\mathrm{G} / \mathrm{G}$ & $233(76.6 \%)$ & $139(70.2 \%)$ & 1 & 0.042 & 6579 & 674.8 \\
\hline & Over-dommant & $\mathrm{A} / \mathrm{G}$ & $71(23.4 \%)$ & $59(29.8 \%)$ & $1.54(1.02-2.34)$ & 0.042 & 051.9 & $0 / 4.0$ \\
\hline & Log-additive & - & - & - & $1.59(1.09-2.32)$ & 0.017 & 656.3 & 673.2 \\
\hline rs10984811 & & $\mathrm{A} / \mathrm{A}$ & $219(72 \%)$ & $139(69.8 \%)$ & 1 & & & \\
\hline & Co-dominant & $\mathrm{C} / \mathrm{A}$ & $80(26.3 \%)$ & $49(24.6 \%)$ & $0.97(0.64-1.49)$ & 0.032 & 659.2 & 680.3 \\
\hline & & $\mathrm{C} / \mathrm{C}$ & $5(1.6 \%)$ & $11(5.5 \%)$ & $3.95(1.33-11.73)$ & & & \\
\hline
\end{tabular}




\begin{tabular}{|c|c|c|c|c|c|c|c|c|}
\hline & Dominant & $\mathrm{A} / \mathrm{A}$ & $219(72 \%)$ & $139(69.8 \%)$ & 1 & 0.52 & 6637 & $68055>$ \\
\hline & Dommant & $\mathrm{C} / \mathrm{A}-\mathrm{C} / \mathrm{C}$ & $85(28 \%)$ & $60(30.1 \%)$ & $1.14(0.76-1.70)$ & 0.02 & 005.1 & 000.3 \\
\hline & & $\mathrm{A} / \mathrm{A}-\mathrm{C} / \mathrm{A}$ & $299(98.4 \%)$ & $188(94.5 \%)$ & 1 & & & \\
\hline & Recessive & $\mathrm{C} / \mathrm{C}$ & $5(1.6 \%)$ & $11(5.5 \%)$ & $3.97(1.34-11.75)$ & 0.0089 & $65 \% .2$ & $6 / 4.1$ \\
\hline & Over-daminant & $\mathrm{A} / \mathrm{A}-\mathrm{C} / \mathrm{C}$ & $224(73.7 \%)$ & $150(75.4 \%)$ & 1 & 068 & 6630 & 6808 \\
\hline & Uver-dominant & $\mathrm{C} / \mathrm{A}$ & $80(26.3 \%)$ & $49(24.6 \%)$ & $0.92(0.60-1.39)$ & 0.68 & 603.9 & 680.8 \\
\hline & Log-additive & - & - & - & $1.28(0.91-1.80)$ & 0.16 & 662 & 678.9 \\
\hline rs17777329 & & $\mathrm{A} / \mathrm{A}$ & $275(90.5 \%)$ & $167(83.9 \%)$ & 1 & & & \\
\hline & Co-dominant & $\mathrm{A} / \mathrm{G}$ & $28(9.2 \%)$ & $30(15.1 \%)$ & $1.88(1.07-3.30)$ & 0.051 & 660.1 & 681.2 \\
\hline & & $\mathrm{G} / \mathrm{G}$ & $1(0.3 \%)$ & $2(1 \%)$ & $3.69(0.33-41.57)$ & & & \\
\hline & Dominant & $\mathrm{A} / \mathrm{A}$ & $275(90.5 \%)$ & $167(83.9 \%)$ & 1 & 0018 & 6584 & 6753 \\
\hline & Domminall & A/G-G/G & $29(9.5 \%)$ & $32(16.1 \%)$ & $1.95(1.12-3.37)$ & 0.010 & 000.4 & $0 / 3.3$ \\
\hline & Recescive & $\mathrm{A} / \mathrm{A}-\mathrm{A} / \mathrm{G}$ & $303(99.7 \%)$ & 197 (99\%) & 1 & 03 & 663 & 6700 \\
\hline & Kecessive & $\mathrm{G} / \mathrm{G}$ & $1(0.3 \%)$ & $2(1 \%)$ & $3.40(0.30-38.26)$ & 0.3 & 003 & $0 / 9.9$ \\
\hline & Oyar dominont & A/A-G/G & $276(90.8 \%)$ & $169(84.9 \%)$ & 1 & 003 & 6503 & 676 \\
\hline & over-comminant & $\mathrm{A} / \mathrm{G}$ & $28(9.2 \%)$ & $30(15.1 \%)$ & $1.87(1.06-3.27)$ & 0.03 & 059.5 & $0 / 0.2$ \\
\hline & Log-additive & - & - & - & $1.89(1.13-3.16)$ & 0.015 & 658.1 & 675 \\
\hline rs 1075355 & & $\mathrm{G} / \mathrm{G}$ & $253(83.2 \%)$ & $149(74.9 \%)$ & 1 & & & \\
\hline & Co-dominant & $\mathrm{G} / \mathrm{C}$ & $46(15.1 \%)$ & $48(24.1 \%)$ & $1.65(1.04-2.62)$ & 0.093 & 661.3 & 682.4 \\
\hline & & $\mathrm{C} / \mathrm{C}$ & $5(1.6 \%)$ & $2(1 \%)$ & $0.71(0.13-3.85)$ & & & \\
\hline & Dominant & $\mathrm{G} / \mathrm{G}$ & $253(83.2 \%)$ & $149(74.9 \%)$ & 1 & & & \\
\hline & Dominant & $\mathrm{G} / \mathrm{C}-\mathrm{C} / \mathrm{C}$ & $51(16.8 \%)$ & $50(25.1 \%)$ & $1.56(1.00-2.45)$ & 0.052 & 660.3 & $6 / 1.2$ \\
\hline & Recescive & G/G-G/C & $299(98.4 \%)$ & 197 (99\%) & 1 & 06 & 6638 & 6807 \\
\hline & hecessive & $\mathrm{C} / \mathrm{C}$ & $5(1.6 \%)$ & $2(1 \%)$ & $0.64(0.12-3.51)$ & 0.0 & 003.0 & 000.1 \\
\hline & & $\mathrm{G} / \mathrm{G}-\mathrm{C} / \mathrm{C}$ & $258(84.9 \%)$ & $151(75.9 \%)$ & 1 & 0032 & 6505 & 6764 \\
\hline & Uver-dominant & $\mathrm{G} / \mathrm{C}$ & $46(15.1 \%)$ & $48(24.1 \%)$ & $1.66(1.04-2.63)$ & 0.032 & 039.5 & $0 / 6.4$ \\
\hline & Log-additive & - & - & - & $1.40(0.93-2.11)$ & 0.1 & 661.4 & 678.3 \\
\hline
\end{tabular}

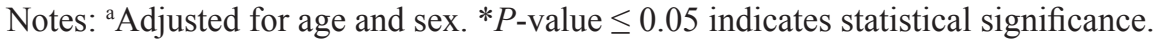

Abbreviations: SNP, single nucleotide polymorphism; OR, odds ratio; CI, confidence interval; AIC, Akaike's Information Criterion; BIC, Bayesian Information Criterion.

modulatory subunits that are electrically silent and cannot form functional homotetramers. These silent subunits form heterotetramers that modulate the properties of other subunits, increasing the functional diversity of channel subfamilies [17]. Voltage-gated $\mathrm{K}^{+}\left(\mathrm{K}_{\mathrm{v}}\right)$ channel activity in pulmonary artery smooth muscle cells (PASMC) is important for the control of apoptosis and proliferation as well as the regulation of membrane potential and pulmonary vascular tone [18]. A previous study demonstrated that KNCV2 contributes to susceptibility to and was considered a genetic modifier of epilepsy [17]. However, the role of KNCV2 in HAPE remains unknown, and additional studies are needed.

The rs 1075355 SNP had the strongest association in this study. It is located in the intron of the VAT1L gene and encodes a vesicle amine transport 1 homologue; its cellular localization and functions have not yet been researched. An association study suggested that a locus on chromosome 16q23-24 (including VAT1L) affected HDLC levels in two independent French-Canadian populations [19]. Additionally, a genome-wide association study of the rate of cognitive decline in Alzheimer's disease indicated that rs 9934540 genetic variants in the VAT1L gene intron were positively associated with the development of Alzheimer's disease [20]. Two different genes, ADAMTS18 and WWOX, are adjacent to the rs1075355 SNP.

ADAMTS18 is a member of the ADAMTS protease family, which is comprised of complex secreted enzymes containing a reprolysin-type prometalloprotease domain attached to an ancillary domain with a highly-conserved structure including at least one thrombospondin type 1 repeat. Known functions of ADAMTS proteases include processing procollagens and von Willebrand factor and catabolism of aggrecan, versican, and brevican. ADAMTS also play important roles in connective tissue organization, 
Table 5: Go and pathway analysis of the top genes of GWAS

\begin{tabular}{|c|c|c|c|}
\hline Function & $p$-value & $\begin{array}{c}\text { Adjusted } \\
p \text {-value }\end{array}$ & Genes \\
\hline zinc ion binding & 7.99E-07 & $1.60 \mathrm{E}-06$ & ADAMTS18;VAT1L \\
\hline protein binding & $1.59 \mathrm{E}-05$ & $1.06 \mathrm{E}-05$ & $I N A D L ; K C N V 2$ \\
\hline thyroxine 5-deiodinase activity & $7.56 \mathrm{E}-05$ & $3.36 \mathrm{E}-05$ & DIO3 \\
\hline very-low-density lipoprotein receptor activity & 2.27E-04 & $6.52 \mathrm{E}-05$ & $V L D L R$ \\
\hline thyroxine 5 '-deiodinase activity & 2.27E-04 & $6.52 \mathrm{E}-05$ & DIO3 \\
\hline metal ion binding & $2.45 \mathrm{E}-04$ & $6.52 \mathrm{E}-05$ & ADAMTS18 \\
\hline low density lipoprotein receptor activity & $8.31 \mathrm{E}-04$ & $1.45 \mathrm{E}-04$ & $V L D L R$ \\
\hline peptidase activity & $8.69 \mathrm{E}-04$ & $1.45 \mathrm{E}-04$ & ADAMTS18 \\
\hline oxidoreductase activity & 0.001153 & $1.58 \mathrm{E}-04$ & $W W O X$ \\
\hline selenium binding & 0.002265 & 2.01E-04 & DIO3 \\
\hline ATP binding & 0.004538 & $3.70 \mathrm{E}-04$ & CCT5 \\
\hline voltage-gated potassium channel activity & 0.007231 & $5.26 \mathrm{E}-04$ & KCNV2 \\
\hline metalloendopeptidase activity & 0.007756 & $5.35 \mathrm{E}-04$ & ADAMTS18 \\
\hline unfolded protein binding & 0.008356 & $5.52 \mathrm{E}-04$ & CCT5 \\
\hline potassium ion binding & 0.00948 & $6.12 \mathrm{E}-04$ & KCNV2 \\
\hline nucleotide binding & 0.010312 & $6.34 \mathrm{E}-04$ & CCT5 \\
\hline manganese ion binding & 0.011276 & $6.63 \mathrm{E}-04$ & NUDT7 \\
\hline coenzyme binding & 0.0115 & 6.67E-04 & WWOX \\
\hline hydrolase activity & 0.012359 & $6.83 \mathrm{E}-04$ & NUDT7 \\
\hline voltage-gated ion channel activity & 0.013964 & 7.35E-04 & KCNV2 \\
\hline protein dimerization activity & 0.031265 & 0.001374 & WWOX \\
\hline magnesium ion binding & 0.032144 & 0.001398 & NUDT7 \\
\hline $\begin{array}{l}\text { hydrolase activity, acting on acid anhydrides, in } \\
\text { phosphorus-containing anhydrides }\end{array}$ & 0.053236 & 0.002117 & NUDT7 \\
\hline calcium ion binding & 0.06698 & 0.002528 & $V L D L R$ \\
\hline receptor activity & 0.121001 & 0.00436 & $V L D L R$ \\
\hline Pathways & $p$-value & $\begin{array}{c}\text { Adjusted } \\
p \text {-value }\end{array}$ & Genes \\
\hline 1,4-Dichlorobenzene degradation & 0.000371 & 0.000742 & CMBL \\
\hline gamma-Hexachlorocyclohexane degradation & 0.006661 & 0.001665 & CMBL \\
\hline Maturity onset diabetes of the young & 0.008871 & 0.001971 & IAPP \\
\hline Tight junction & 0.049301 & 0.002641 & INADL \\
\hline Wnt signaling pathway & 0.054948 & 0.002641 & PPP2R5C \\
\hline
\end{tabular}

coagulation, inflammation, arthritis, angiogenesis, and cell migration $[21,22]$ and are regulated by the Tissue Inhibitor of Metalloproteinase 3 Gene (TIMP3). Furthermore, Kobayashi et al.'s study in a Japanese population demonstrated that TIMP3 was associated with susceptibility to HAPE [23]. TIMPs play a crucial role in the physiological turnover of the extracellular matrix (ECM) by tightly regulating matrix metalloproteinase (MMP) activity [24]. TIMP3 is the only TIMP that binds tightly to the ECM, and the balance between MMPs and
TIMPs plays an important role in maintaining the integrity of healthy tissues. Disturbances of the TIMP/MMP system are implicated in various pathologic conditions in lungs, including pulmonary inflammation, edema, emphysema, and fibrosis, where loss of ECM integrity is a principal feature [25]. Our findings together with those of previous studies demonstrate that the balance between MMPs and TIMPs plays an important role in HAPE pathogenesis.

The human WWOX gene encodes a putative tumor suppressor WW domain-containing oxidoreductase WOX1 
(also known as WWOX or FOR). High frequencies of loss of heterozygosity (LOH) in this gene have been observed in prostate, lung, breast, and other cancers [27]. A recent genome-wide association analysis identified WWOX as one of the loci associated with forced vital capacity (FVC), a spirometric measure of pulmonary function used to diagnose and monitor lung diseases [27]. These findings indicate that the WWOX gene may be involved in lung development and the pathogenesis of restrictive lung disease; future studies are needed to determine whether WWOX is similarly associated with HAPE pathogenesis.

Although the statistical power of the present study was sufficient, some limitations should be considered when interpreting these results. First, the patient sample sizes were relatively small, and the association between the identified polymorphisms and HAPE susceptibility should be confirmed in future studies with larger sample sizes. Secondly, the mechanisms by which the potential candidate genes contribute to the pathogenesis of HAPE remain unclear, and functional studies of these candidate genes are needed.In conclusion, our study provides new evidence regarding the pathogenesis of HAPE in the Han Chinese population. Although the genetic factors that contribute to the development of HAPE remain largely unknown, we identified candidate genes that contribute to HAPE susceptibility. However, polymorphisms in these genes should be examined further before definitive conclusions regarding their role in HAPE pathogenesis can be made.

\section{MATERIALS AND METHODS}

\section{Study populations}

In this two-stage case-control study, we evaluated associations between genetic variants across the human genome and the risk of HAPE. All participants included in the study were from the Han Chinese population. Study subjects for both GWAS scan of HAPE and the replication phase of the experiment were selected according to detailed inclusion and exclusion criteria. Briefly, patients who lived on the Tibet Plateau and were diagnosed with HAPE were recruited from the General Hospital of Tibet Military Region. Control subjects were Han Chinese immigrants living in Lhasa, Tibet, and their medical histories and physical examinations confirmed that they were in good health. Demographic information was collected through interviews using a standard questionnaire. Ultimately, 267 HAPE cases (89 recurrent HAPE cases; mean age $32.6 \pm 10.7$ years) and 304 controls (mean age $36.2 \pm 4.5$ years) were selected for the study. Two $\mathrm{mL}$ of venous blood were collected from each individual into tubes containing 2\% EDTA-K2, centrifuged, and stored at $-80^{\circ} \mathrm{C}$ until analysis. DNA was extracted from whole blood samples using the QIAamp ${ }^{\circledR}$ DNA Blood Mini kit (Qiagen), and DNA concentrations were measured using a NanoDrop 2000. Informed consent was obtained from all subjects, and the Human Ethics Committee of our institute approved the investigation.

\section{Study design}

For the GWAS scan experiment, we scanned DNA samples from 68 patients with recurrent HAPE using Affymetrix SNP Array 6.0 Chips. The allele frequencies of the 68 patients were then compared to those of 84 HapMap CHB subjects to identify significant differences in SNP frequencies. In the replication experiment, associations between the SNPs identified in the GWAS scan and risk of HAPE where examined in 199 HAPE patients and 304 unrelated healthy controls. Furthermore, to identify candidate genes that might underlie HAPE susceptibility, we conducted Gene Ontology (GO) and Kyoto Encyclopedia of Genes and Genomes (KEGG) pathway enrichment analysis for the genes involved in the associated genetic loci.

\section{Quality control (QC) in GWAS}

A total of 906,660 SNPs were genotyped in 68 patients with recurrent HAPE during the GWAS experiment using Affymetrix Genome-Wide Human SNP Array 6.0 Chips as described previously [28]. A systematic quality control (QC) procedure was applied to both SNPs and samples prior to the association analysis. SNPs were excluded if they (i) did not map onto autosomal chromosomes; (ii) had a call rate of less than $95 \%$; (iii) had a minor allele frequency (MAF) less than 0.05 ; or (iv) deviated from Hardy-Weinberg equilibrium $(p<0.001)$. Sixty-eight HAPE cases and 84 controls with 502,689 SNPs remained after QC.

\section{SNP selection and genotyping in the replication study}

After genome-wide association analysis, we compared the allele frequencies of the 502,689 SNPs in the 68 recurrent HAPE cases to those in the 84 HapMap CHB controls using a chi-squared $\left(\chi^{2}\right)$ test. Allele frequencies differed significantly between HAPE cases and controls for 77 SNPs. In the replication study, these 77 SNPs were genotyped in 199 HAPE patients and 304 normal controls. SNPs that were significantly associated with HAPE risk $(p<0.05)$ in the replication study were selected for GO and KEGG pathway enrichment analyses. Genotyping was performed using Sequenom MassARRAY Assay Design 3.0 Software [29] with a genotype success rate greater than $97.3 \%$.

\section{Statistical analysis}

SPSS 17.0 statistical software was used for statistical analysis. An exact test was used to test the departure of each SNP frequency from Hardy-Weinberg equilibrium 
(HWE) in control subjects. Differences in SNP genotype distribution between HAPE patients and controls were compared using a $\chi^{2}$ test [30]. Odds ratios (ORs) and $95 \%$ confidence intervals (CIs) were determined using unconditional logistic regression analysis with adjustments for age and gender [31]. All $p$ values presented in this study are two-sided; $p<0.05$ indicated a statistically significant difference.

Associations between SNPs and HAPE risk were tested using various genetic models (co-dominant, dominant, over-dominant, recessive, and log-additive) and analyzed using SNP Stats software (obtained from http:// bioinfo.iconcologia.net, Catalan Institute of Oncology, Barcelona, Spain). To reduce population stratification, a multidimensional scaling approach (MDS) was used and a QQ-plot was generated using PLINK software (version 1.07) (http://www.cog-genomics.org/plink2/) [32]. R software (version 2.11.1) was used for statistical analysis and to generate plots, including Manhattan plots. GO analysis were performed using Bingo software [33], and pathway enrichment analyses were performed using Mas 3.0 software (http://bioinfo.capitalbio.com/mas3/).

\section{Authors' contributions}

Not applicable.

\section{ACKNOWLEDGMENTS}

We are grateful to all of the patients and other individuals who made this work possible. We would also like to thank the clinicians and hospital staff who contributed to data collection for this study.

\section{CONFLICTS OF INTEREST} interests

The authors declare that they have no competing

\section{FUNDING}

This work was supported by the Science and Technology Project of Tibet Autonomous Region (2009Z3 ) and by a general financial grant from the China Postdoctoral Science Foundation (2012M512186).

\section{REFERENCES}

1. Shrestha P, Pun M, Basnyat B. High altitude pulmonary edema (HAPE) in a Himalayan trekker: a case report. Extreme physiology \& medicine. 2014; 3:6.

2. Maggiorini M. Prevention and treatment of high-altitude pulmonary edema. Prog Cardiovasc Dis. 2010; 52:500-506.

3. Sartori C, Allemann Y, Trueb L, Lepori M, Maggiorini M, Nicod P, Scherrer U. Exaggerated pulmonary hypertension is not sufficient to trigger high-altitude pulmonary oedema in humans. Schweiz Med Wschr. 2000; 130:385-389.

4. West JB, Colice GL, Lee YJ, Namba Y, Kurdak SS, Fu Z, Ou LC, Mathieu-Costello O. Pathogenesis of high-altitude pulmonary oedema: direct evidence of stress failure of pulmonary capillaries. Eur Respir J. 1995; 8:523-529.

5. Bartsch P. High altitude pulmonary edema. Respiration; international review of thoracic diseases. 1997; 64:435-443.

6. Sartori C, Trueb L, Scherrer U. High-altitude pulmonary edema. Mechanisms and management. Cardiologia (Rome, Italy). 1997; 42:559-567.

7. Bartsch P, Maggiorini M, Ritter M, Noti C, Vock P, Oelz O. Prevention of high-altitude pulmonary edema by nifedipine. New Engl J Med. 1991; 325:1284-1289.

8. Zhang D, Zhou Q, Yang J. Epidemic Charaeters of Acute High Altitude Pulmonary Edema in High Altitude Areas. West China Medical Journal. 2001; 16:316-317.

9. Bhagi S, Srivastava S, Singh SB. High-altitude pulmonary edema: review. Int J Occup Med Env. 2014; 56:235-243.

10. Mortimer H, Patel S, Peacock AJ. The genetic basis of highaltitude pulmonary oedema. Pharmacology \& therapeutics. 2004; 101:183-192.

11. Stobdan T, Kumar R, Mohammad G, Thinlas T, Norboo T, Iqbal M, Pasha MA. Probable role of beta2-adrenergic receptor gene haplotype in high-altitude pulmonary oedema. Respirology (Carlton, Vic). 2010; 15:651-658.

12. Hanaoka M, Droma Y, Ota M, Ito M, Katsuyama Y, Kubo K. Polymorphisms of human vascular endothelial growth factor gene in high-altitude pulmonary oedema susceptible subjects. Respirology (Carlton, Vic). 2009; 14:46-52.

13. Hotta J, Hanaoka M, Droma Y, Katsuyama Y, Ota M, Kobayashi T. Polymorphisms of renin-angiotensin system genes with high-altitude pulmonary edema in Japanese subjects. Chest. 2004; 126:825-830.

14. Saxena S, Kumar R, Madan T, Gupta V, Muralidhar K, Sarma PU. Association of polymorphisms in pulmonary surfactant protein A1 and A2 genes with high-altitude pulmonary edema. Chest. 2005; 128:1611-1619.

15. Chen Z, Leibiger I, Katz AI, Bertorello AM. Pals-associated tight junction protein functionally links dopamine and angiotensin II to the regulation of sodium transport in renal epithelial cells. Brit J Pharmacol. 2009; 158:486-493.

16. Shin K, Straight S, Margolis B. PATJ regulates tight junction formation and polarity in mammalian epithelial cells. J Cell Biol. 2005; 168:705-711.

17. Jorge BS, Campbell CM, Miller AR, Rutter ED, Gurnett CA, Vanoye CG, George AL Jr, Kearney JA. Voltage-gated potassium channel KCNV2 (Kv8.2) contributes to epilepsy susceptibility. Proc Natl Acad Sci U S A. 2011; 108:5443-5448.

18. Fantozzi I, Platoshyn O, Wong AH, Zhang S, Remillard CV, Furtado MR, Petrauskene OV, Yuan JX. Bone morphogenetic protein-2 upregulates expression and function of voltagegated $\mathrm{K}+$ channels in human pulmonary artery smooth muscle cells. Am J Physiol-Lung C. 2006; 291:L993-1004. 
19. Dastani Z, Pajukanta P, Marcil M, Rudzicz N, Ruel I, Bailey SD, Lee JC, Lemire M, Faith J, Platko J, Rioux J, Hudson TJ, Gaudet D, et al. Fine mapping and association studies of a high-density lipoprotein cholesterol linkage region on chromosome 16 in French-Canadian subjects. Eur J Hum Genet. 2010; 18:342-347.

20. Sherva R, Tripodis Y, Bennett DA, Chibnik LB, Crane PK, de Jager PL, Farrer LA, Saykin AJ, Shulman JM, Naj A, Green RC. Genome-wide association study of the rate of cognitive decline in Alzheimer's disease. Alzheimer's \& dementia. 2014; 10:45-52.

21. Cal S, Obaya AJ, Llamazares M, Garabaya C, Quesada V, Lopez-Otin C. Cloning, expression analysis, and structural characterization of seven novel human ADAMTSs, a family of metalloproteinases with disintegrin and thrombospondin-1 domains. Gene. 2002; 283:49-62.

22. Apte SS. A disintegrin-like and metalloprotease (reprolysin type) with thrombospondin type 1 motifs: the ADAMTS family. Int J Biochem Cell B. 2004; 36:981-985.

23. Kobayashi N, Hanaoka M, Droma Y, Ito M, Katsuyama Y, Kubo K, Ota M. Polymorphisms of the tissue inhibitor of metalloproteinase 3 gene are associated with resistance to high-altitude pulmonary edema (HAPE) in a Japanese population: a case control study using polymorphic microsatellite markers. PLoS One. 2013; 8:e71993.

24. Loffek S, Schilling O, Franzke CW. Series "matrix metalloproteinases in lung health and disease": Biological role of matrix metalloproteinases: a critical balance. Eur Respir J. 2011; 38:191-208.

25. Clark IM, Swingler TE, Sampieri CL, Edwards DR. The regulation of matrix metalloproteinases and their inhibitors. Int J Biochem Cell B. 2008; 40:1362-1378.
26. Chang NS, Doherty J, Ensign A, Lewis J, Heath J, Schultz L, Chen ST, Oppermann U. Molecular mechanisms underlying WOX1 activation during apoptotic and stress responses. Biochem Pharmacol. 2003; 66:1347-1354.

27. Loth DW, Artigas MS, Gharib SA, Wain LV, Franceschini N, Koch B, Pottinger TD, Smith AV, Duan Q, Oldmeadow C, Lee MK, Strachan DP, James AL, et al. Genome-wide association analysis identifies six new loci associated with forced vital capacity. Nat Genet. 2014; 46:669-677.

28. Hu Z, Wu C, Shi Y, Guo H, Zhao X, Yin Z, Yang L, Dai J, Hu L, Tan W, Li Z, Deng Q, Wang J, et al. A genomewide association study identifies two new lung cancer susceptibility loci at 13q12.12 and 22q12.2 in Han Chinese. Nat Genet. 2011; 43:792-796.

29. Gabriel S, Ziaugra L, Tabbaa D. SNP genotyping using the Sequenom MassARRAY iPLEX platform. Current protocols in human genetics. 2009; Chapter 2:Unit 2.12.

30. Adamec C. [Example of the use of the nonparametric test.test X2 for comparison of 2 independent examples]. [Article in Czech]. Ceskoslovenske zdravotnictvi. 1964; 12: 613-619.

31. Bland JM, Altman DG. The odds ratio. Bmj. 2000; 320:1468.

32. Purcell S, Neale B, Todd-Brown K, Thomas L, Ferreira MA, Bender D, Maller J, Sklar P, de Bakker PI, Daly MJ, Sham PC. PLINK: a tool set for whole-genome association and population-based linkage analyses. Am J Hum Genet. 2007; 81:559-575.

33. Liu M, Wu B, Wang WZ, Lee LM, Zhang SH, Kong LZ. Stroke in China: epidemiology, prevention, and management strategies. Lancet Neurol. 2007; 6:456-464. 